\title{
Determining the number of factors to retain: A Windows-based FORTRAN-IMSL program for parallel analysis
}

\author{
JENNIFER D. KAUFMAN and WILIIAM P. DUNLAP \\ Thlane University, New Orleans, Louisiana
}

\begin{abstract}
Parallel analysis (PA; Horn, 1965) is a technique for determining the number of factors to retain in exploratory factor analysis that has been shown to be superior to more widely known methods (Zwick \& Velicer, 1986). Despite its merits, PA is not widely used in the psychological literature, probably because the method is unfamiliar and because modern, Windows-compatible software to perform PA is unavailable. We provide a FORTRAN-IMSL program for PA that runs on a PC under Windows; it is interactive and designed to suit the range of problems encountered in most psychological research. Furthermore, we provide sample output from the PA program in the form of tabled values that can be used to verify the program operation; or, they can be used either directly or with interpolation to meet specific needs of the researcher
\end{abstract}

Selecting the correct number of factors to retain in exploratory factor analysis is of vital importance to researchers as an aid in the measurement and interpretation of psychological constructs (Cota, Longman, Holden, Fekken, \& Xinaris, 1993). The consequences of failure in selecting the "correct" number of factors may result in serious distortion of reported research results (Cota et al., 1993). Although factor analysis is an important tool of many researchers in the psychological sciences, the methods used by most researchers to determine the number of factors to retain are less than optimal.

To date, Kaiser's (1960) rule is the most common method used in determining the number of factors (Lautenschlager, 1989). Kaiser's rule is simply to retain factors whose eigenvalues are greater than 1 . Kaiser's rule is based on the assumption that to retain a factor that explains less variance than a single original variable is not psychometrically reasonable. Despite the fact that Kaiser's "rule of thumb" is both simple and widely applied in psychological research, it is often criticized. For example, application of Kaiser's rule suggests that an eigenvalue of 1.01 represents a meaningful factor whereas an eigenvalue of .99 does not. But using a rule that dictates a strict criterion eigenvalue of 1.00 regardless of the data or constructs being measured is overly simplistic.

In addition to Kaiser's rule, another common method used in factor analysis is the scree test (Cattell, 1966). The scree test requires a plot of the obtained eigenvalues that is then subjected to visual inspection and interpretation. The researcher looks for a discontinuity in the plot of the eigenvalues that separates meaningful factors or compo-

Correspondence should be addressed to W. D. Dunlap, Department of Psychology, Tulane University, New Orleans, LA 70118 (e-mail: dunlap@ tulane.edu). nents from the "scree" or the remaining inconsequential values. The scree test rests on the assumption that a researcher's visual inspection will clearly distinguish the discontinuity in the plot that separates eigenvalues that delineate major factors from those that represent noise or scree. In practice, visual interpretation of scree plots is inherently subjective and has been criticized by researchers who often have difficulty locating discontinuities in the plots (Turner, 1998).

Although there are other options for factor determination, such as Lawley and Maxwell's (1963) maximumlikelihood factor method of factor extraction, Kaiser's rule and the scree plot are the techniques most frequently reported in the literature. One desirable alternative to Kaiser's rule and the scree plot is a factor-analytic technique referred to as parallel analysis (PA; Horn, 1965). To date, PA has shown the most promising results as a method for determining the correct number of factors to retain in factor analysis (Fabrigar, Wegener, MacCallum, \& Strahan, 1999; Humphreys \& Montanelli, 1975; Zwick \& Velicer, 1986). For example, Zwick and Velicer showed that PA recovered the correct number of factors in simulated data more accurately than other methods. An example is provided in Figure 1. Data representing 369 respondents and 44 variables were taken from a widely used multivariate textbook (Tabachnick \& Fidell, 1996). The scree plot depicts the actual eigenvalues from the data as well as using both Kaiser's rule and parallel analysis. As can be seen from viewing the scree plot, a judgment can be made at the break in the plotted values somewhere between the 3rd and 6th eigenvalues, whereas parallel analysis clearly suggests keeping 5 factors, and Kaiser's rule suggests retaining 12 factors.

Despite the merits of PA, very little published factoranalytic research reports application of this factordetermining technique. We contend that PA is used so 


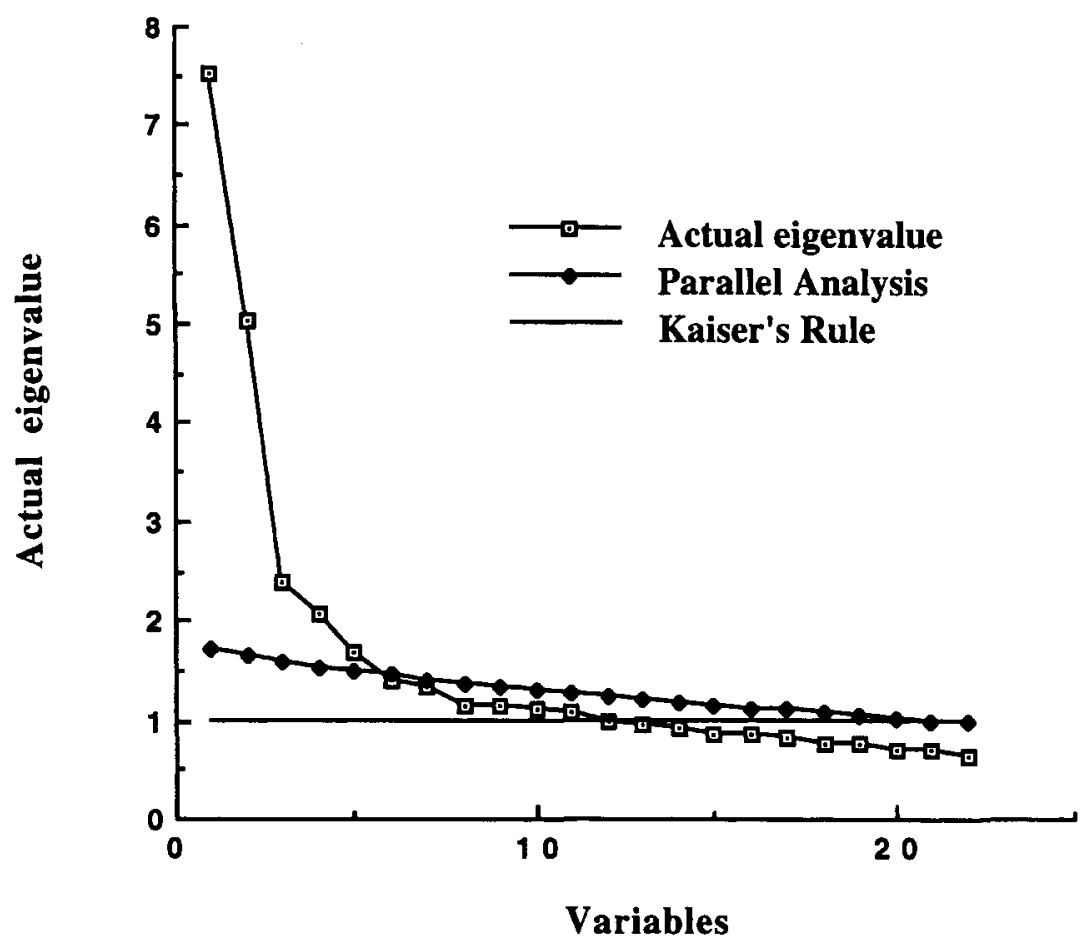

Figure 1. Plot of actual eigenvalues, parallel analysis values, and Kaiser's rule of 1 using data from Tabachnick and Fidell (1996).

seldom, at least in part, because of the inaccessibility of programs that perform PA. None of the commonly used existing software packages (i.e., SAS, SPSS, BMDP) have been equipped to handle PA, though O'Connor (2000) has provided a tutorial on how to use PA with SPSS and SAS. In addition, we could find very few readily available, easyto-use PA computer programs described in the current literature. The only operational existing PA program in the literature is now 10 years old (Longman, Cota, Holden, \& Fekken, 1989). The logic of Longman et al.'s program is parallel to that used in the development of the present program. Although the program by Longman et al. provides accurate values, its lack of current popularity may be a result of requiring the researcher to have knowledge of FORTRAN and ability to compile FORTRAN programs. Therefore, the purpose of the present article and program is to build on and improve Longman et al.'s program and provide researchers with a simple, updated, faster, and Windows-based personal computer program to be used for conducting PA.

Specifically, our PA program is simpler and faster than that provided by Longman et al. (1989) in the following ways: (1) Longman et al.'s program requires the researcher to change the program parameters, whereas the present program is interactive; (2) Longman et al.'s program must be recompiled each time a problem is run and the output is sent to "unit 7," whereas ours appears on the researcher's computer monitor; (3) Longman et al.'s program requires the researcher to set the seed for random number generation, whereas ours uses an internal seed;
(4) although Longman et al.'s program uses doubleprecision subroutines, and the current program uses single-precision subroutines, with 5-6 decimal place accuracy that single precision provides being more than adequate for the accuracy of the Monte Carlo approximations; and (5) although Longman et al.'s program stores and sorts the eigenvalue cutoffs at differing probability levels, this feature slows the program and requires more room for storage. The present program, presented in the Appendix, offers mean eigenvalues based on 1,000 iterations. However, if percentile information is desired, an alternative program is also provided that computes percentile results for eigenvalues specified by the user; and finally, (6) unlike Longman et al.'s program, the present program may be run in a Windows-based environment, and both programs may be sent to the researcher as compiled programs rather than requiring a FORTRAN compiler or the IMSL subroutine package and the knowledge required to use it.

In the present article we not only provide an updated program for PA but also present tables of PA values for a range of sample sizes and numbers of variables that covers a substantial proportion of most psychological research needs. The following section provides a brief overview and demonstration of PA.

\section{Parallel Analysis}

PA involves a Monte Carlo approximation of the expected values of ordered eigenvalues that would result from random independent normal variates given the sam- 
ple size and the original number of variables. The values are approximate because no closed mathematical solution to these values is known. The rationale assumes that meaningful components extracted from actual data will have larger eigenvalues than eigenvalues obtained from random normal variates generated with the same sample size and number of variables (Lautenschlager, 1989). Several articles have offered regression equations to be used for approximating the expected eigenvalues used as PA criteria. Some of the earliest work was done by Montanelli and Humphreys (1976), who placed squared multiple correlations of the correlation matrix analyzed on the diagonal, which is the first-step approximation to principal axis factor analysis. In order to determine the number of factors to retain, Montanelli and Humphreys plotted the eigenvalues from the random and actual correlation matrices on the same axes. The point at which the curves cross indicates the number of factors to retain. This "crossover" point separates meaningful factors from noise factors because it is assumed that a researcher will not be interested in a factor that accounts for less variance than the same factor obtained from a matrix of random independent normal data.

A decade later, Allen and Hubbard (1986) modified Montanelli and Humphreys's (1976) approach by applying PA to principal component analysis using 1s rather than squared multiple $R \mathrm{~s}$ on the matrix diagonal. Lautenschlager, Lance, and Flaherty (1989) then provided revised regression equations that were superior to those of Allen and Hubbard because they made more exact estimates of the first eigenvalue. This improvement is based on the fact that the regression equations are recursive; initial eigenvalues affect estimates of later eigenvalues.

Lautenschlager (1989) subsequently generated tables from these improved equations and lifted the restriction that eigenvalues must be estimated with a specific $N$ (number of subjects), and $p$ (number of variables) combination by providing linear interpolation methods to estimate nontabled $N$ and $p$ combinations. The approach in the present article is to avoid both the problem of using complex regression equations and fixed values of $N$ and $p$ by providing a program that directly estimates expected eigenvalues for the needed values of $N$ and $p$ entered interactively by the user.

Despite the advances in the development of PA, application of this superior method of factor determination has been limited (Fabrigar et al., 1999). Lautenschlager's (1989) tables are based on sample sizes $(\mathrm{Ns})$ ranging from 50 to 2,000 and $p$ s ranging from 5 to 80 . Although the choice of such values mirrors the sentiments of many researchers who have suggested that samples used in factor analysis should consist of 500 or more subjects (Comrey \& Lee, 1992), and an $N: p$ ratio of at least 10 (Everitt, 1975), in practice such conservative rules are often ignored. Furthermore, MacCallum, Widaman, Zhang, and Hong (1999) showed that rules of thumb such as these regarding sample size in factor analysis are not always valid. MacCallum et al. suggested that the smallest $N$, or the minimum $N: p$ ratio needed to ensure "good recovery of population factors is not constant across studies, but rather, is dependent on certain aspects of the variables and design in a given study" (p. 96).

In practice, factor analysis is often based on much smaller $N$ and $p$ combinations than the recommended rules of thumb discussed above. Fabrigar et al. (1999) surveyed two top-tier psychology journals, the Journal of Personality and Social Psychology (JPSP) and the Journal of Applied Psychology (JAP) from 1991 to 1995. The authors concluded that exploratory factor analysis continues to be an extremely popular statistical procedure in psychological research.

They found that a total of 159 of the 883 articles published in JPSP and a total of 58 of 455 articles published in $J A P$ reported the use of exploratory factor analysis. Thus, the typical issue of these journals contained two or three articles using exploratory factor analysis. Despite the popularity of exploratory factor analysis, $P A$ was used in only a single article in 5 years' publication of these two top-tier journals. We also surveyed JAP and another social psychology journal (Personality and Social Psychology Bulletin) for a single year, 1998. Consistent with the findings reported by Fabrigar et al. (1999), we found that exploratory factor analysis was used in 23 articles; however, none of the 23 reported the use of $P A$. In addition to noting the method of factor extraction used, we also recorded information on the mean, median, and standard deviation of the number of subjects used in these analyses. We found mean $N=644$, median $N=180$, and the $S D=1,700$. The mean number of variables was 14 , the median was 10 , and the standard deviation was 11 . The average number of identified factors was 2 , the median was 2 , and the standard deviation was 1 . Our brief survey of the psychological literature revealed that about $23 \%$ of factor-analytic results were based on fewer than 100 subjects, and more than half of all reported factor analyses were based on samples of \pm 180 . More than half of the studies were based on fewer than 10 variables, and $83 \%$ of the studies resulted in the identification of 2 or fewer factors ( $43 \%$ identified a single factor).

Thus, the results of our survey suggest that much exploratory factor-analytic research is based on relatively small numbers of subjects, variables, and factors. Therefore, one goal of the present article is to provide a table of chance eigenvalues that covers smaller sample sizes common to much psychological research. On the basis of our preliminary survey, we present tabled values including $N$ s ranging from 30 to 200 , in increments of 10 . The tables also contain values for $p$ ranging from 5 to 15 in increments of 5. In order to ensure the accuracy of our tabled estimates, all reported values are averaged from 1,000 Monte Carlo iterations.

A second goal of this article is to provide researchers with an interactive and easy-to-use PA program. It is our hope that the tabled values will meet the needs of many researchers; however, we realize that other $N$ and $p$ combinations will be needed. In order to estimate chance eigenvalues for specific $N$ and $p$ combinations not provided in the tables, one alternative is to perform linear in- 
Table 1

Table of Eigenvalues From 1,000 Monte Carlo Simulations for $N=30-200$ and $p=5$

\begin{tabular}{cccccc}
\hline & \multicolumn{5}{c}{ Variables $(p)$} \\
\cline { 2 - 6 } Subjects $(N)$ & 1 & 2 & 3 & 4 & 5 \\
\hline 30 & 1.542 & 1.209 & .972 & .751 & .526 \\
40 & 1.467 & 1.183 & .977 & .786 & .587 \\
50 & 1.407 & 1.165 & .987 & .814 & .626 \\
60 & 1.374 & 1.151 & .987 & .832 & .657 \\
70 & 1.349 & 1.139 & .987 & .842 & .683 \\
80 & 1.323 & 1.131 & .990 & .855 & .701 \\
90 & 1.306 & 1.124 & .991 & .864 & .715 \\
100 & 1.286 & 1.114 & .994 & .873 & .732 \\
110 & 1.274 & 1.112 & .991 & .877 & .744 \\
120 & 1.262 & 1.107 & .992 & .883 & .756 \\
130 & 1.251 & 1.102 & .993 & .889 & .764 \\
140 & 1.244 & 1.104 & .993 & .890 & .770 \\
150 & 1.230 & 1.096 & .995 & .896 & .782 \\
160 & 1.227 & 1.094 & .993 & .899 & .787 \\
170 & 1.218 & 1.091 & .995 & .904 & .793 \\
180 & 1.211 & 1.087 & .994 & .907 & .801 \\
190 & 1.208 & 1.085 & .995 & .907 & .804 \\
200 & 1.201 & 1.084 & .995 & .909 & .811 \\
\hline
\end{tabular}

terpolation based on the values that are provided. Another alternative is to compute the estimated values directly by entering the specific $N: p$ combination into a PA program. Thus, we provide a simple, interactive FORTRAN PA computer program available as source code or as a compiled version that runs under PC Windows ' 95 and higher. We believe that our PA program is simple to use and that it will accommodate research problems with samples as large as 1,000 and as many as 50 variables; the source code can also be modified to fit larger requirements.

\section{Tables and PA Program}

For the tables, expected ordered eigenvalues were generated in a series of Monte Carlo simulations in which the number of variables $(p)$ included 5, 10, and 15; sample sizes $(N)$ ranged from 30 to 200 in increments of 10 ; and 1,000 iterations were used for each point. The program inputs a particular $N$ and $p$ combination and generates random normal data matrices using subroutines from the International Mathematical and Statistical Libraries (IMSL, 1982). IMSL was used to generate random data for $N$ cases on each of $p$ independent variables from an $N(0,1)$ population, create a correlation matrix based on these data, and compute the eigenvalues of each correlation matrix. Output of the program described has been checked by comparing the values to existing tables (Lautenschlager, 1989) and the values differ by at most 1 point in the second decimal place due to rounding (e.g., 1.392 vs. 1.407). Because our values are based on more Monte Carlo replications, we feel they are likely more accurate than previous tabled values. On average, the eigenvalues obtained using our program differ at most by approximately 2 points in the third decimal place when multiple runs of the same problem are compared.

The FORTRAN source code for this PA program, written to estimate eigenvalues for specific $N$ and $p$ combinations, is presented in the Appendix. Sample output from the PA program is presented in Tables $1-3$. The program takes less than $5 \mathrm{sec}$ to compute smaller problems (e.g., $N<100, p=5$ ), and approximately $30 \mathrm{sec}$ to produce output for larger $N$ and $p$ combinations (e.g., $N=$ $500, p=10$ ).

\section{Discussion}

A recent multivariate text by Sharma (1996) recommends the use of PA as the best way of determining the number of factors; however, this text recommends application of the Allen and Hubbard (1986) regression equations. We feel that because such equations are difficult for researchers to use, despite the merits of PA, the psy-

Table 2

Table of Eigenvalues From 1,000 Monte Carlo Simulations for $N=30-200$ and $p=10$

\begin{tabular}{|c|c|c|c|c|c|c|c|c|c|c|}
\hline \multirow[b]{2}{*}{ Subjects $(N)$} & \multicolumn{10}{|c|}{ Variables $(p)$} \\
\hline & 1 & 2 & 3 & 4 & 5 & 6 & 7 & 8 & 9 & 10 \\
\hline 30 & 2.048 & 1.673 & 1.408 & 1.191 & .999 & .825 & .672 & .528 & .395 & .260 \\
\hline 40 & 1.873 & 1.578 & 1.352 & 1.172 & 1.015 & .868 & .727 & .597 & .474 & .342 \\
\hline 50 & 1.778 & 1.514 & 1.324 & 1.157 & 1.017 & .883 & .761 & .643 & .524 & .400 \\
\hline 60 & 1.695 & 1.464 & 1.294 & 1.149 & 1.019 & .902 & .789 & .678 & .565 & .444 \\
\hline 70 & 1.649 & 1.432 & 1.278 & 1.141 & 1.019 & .906 & .802 & .697 & .594 & .482 \\
\hline 80 & 1.594 & 1.405 & 1.259 & 1.131 & 1.020 & .918 & .821 & .721 & .621 & .510 \\
\hline 90 & 1.560 & 1.374 & 1.244 & 1.126 & 1.023 & .924 & .830 & .737 & .644 & .537 \\
\hline 100 & 1.528 & 1.59 & 1.232 & 1.118 & 1.021 & .929 & .842 & .752 & .662 & .557 \\
\hline 110 & 1.507 & 1.342 & 1.220 & 1.117 & 1.020 & .933 & .847 & .769 & .669 & .574 \\
\hline 120 & 1.483 & 1.330 & 1.212 & 1.112 & 1.024 & .939 & .855 & .771 & .686 & .575 \\
\hline 130 & 1.459 & 1.310 & 1.202 & 1.107 & 1.020 & .943 & .863 & .786 & .703 & .608 \\
\hline 140 & 1.443 & 1.301 & 1.194 & 1.104 & 1.023 & .945 & .868 & .792 & .711 & .619 \\
\hline 150 & 1.429 & 1.291 & 1.190 & 1.101 & 1.023 & .942 & .874 & .799 & .721 & .629 \\
\hline 160 & 1.411 & 1.281 & 1.183 & 1.099 & 1.021 & .949 & .877 & .807 & .729 & .642 \\
\hline 170 & 1.402 & 1.272 & 1.178 & 1.099 & 1.019 & .952 & .880 & .811 & .737 & .651 \\
\hline 180 & 1.387 & 1.266 & 1.174 & 1.093 & 1.021 & .953 & .885 & .818 & .743 & .659 \\
\hline 190 & 1.378 & 1.255 & 1.167 & 1.090 & 1.020 & .955 & .889 & .824 & .753 & .669 \\
\hline 200 & 1.366 & 1.250 & 1.163 & 1.089 & 1.020 & .955 & .891 & .828 & .760 & .677 \\
\hline
\end{tabular}


Table 3

Table of Eigenvalues From 1,000 Monte Carlo Simulations for $N=30-200$ and $p=15$

\begin{tabular}{ccccccccccc}
\hline & \multicolumn{10}{c}{ Variables $(p)$} \\
\cline { 2 - 12 } Subjects $(N)$ & 1 & 2 & 3 & 4 & 5 & 6 & 7 & 8 & 9 & 10 \\
\hline 30 & 2.448 & 2.066 & 1.782 & 1.544 & 1.341 & 1.164 & .999 & .854 & .720 & .596 \\
40 & 2.230 & 1.897 & 1.671 & 1.475 & 1.312 & 1.160 & 1.023 & .892 & .775 & .665 \\
50 & 2.075 & 1.804 & 1.601 & 1.428 & 1.282 & 1.149 & 1.029 & .917 & .808 & .711 \\
60 & 1.966 & 1.724 & 1.547 & 1.396 & 1.263 & 1.140 & 1.031 & .931 & .832 & .742 \\
70 & 1.883 & 1.666 & 1.507 & 1.369 & 1.246 & 1.137 & 1.036 & .940 & .850 & .764 \\
80 & 1.822 & 1.617 & 1.470 & 1.344 & 1.23 & 1.132 & 1.039 & .947 & .866 & .784 \\
90 & 1.771 & 1.579 & 1.442 & 1.322 & 1.220 & 1.128 & 1.038 & .953 & .877 & .799 \\
100 & 1.727 & 1.556 & 1.421 & 1.307 & 1.213 & 1.122 & 1.038 & .957 & .882 & .807 \\
110 & 1.688 & 1.522 & 1.398 & 1.295 & 1.202 & 1.116 & 1.038 & .963 & .892 & .822 \\
120 & 1.657 & 1.501 & 1.384 & 1.283 & 1.196 & 1.114 & 1.037 & .966 & .896 & .831 \\
130 & 1.627 & 1.478 & 1.365 & 1.271 & 1.188 & 1.111 & 1.038 & .968 & .904 & .840 \\
140 & 1.601 & 1.462 & 1.353 & 1.263 & 1.182 & 1.108 & 1.038 & .970 & .906 & .843 \\
150 & 1.580 & 1.438 & 1.342 & 1.253 & 1.176 & 1.105 & 1.037 & .973 & .912 & .851 \\
160 & 1.561 & 1.427 & 1.328 & 1.244 & 1.169 & 1.100 & 1.036 & .974 & .914 & .856 \\
170 & 1.543 & 1.412 & 1.321 & 1.237 & 1.167 & 1.101 & 1.037 & .976 & .918 & .863 \\
180 & 1.525 & 1.404 & 1.312 & 1.234 & 1.163 & 1.097 & 1.035 & .977 & .921 & .866 \\
190 & 1.506 & 1.389 & 1.301 & 1.225 & 1.157 & 1.095 & 1.037 & .979 & .923 & .871 \\
200 & 1.497 & 1.381 & 1.294 & 1.218 & 1.157 & 1.093 & 1.035 & .980 & .926 & .874 \\
\hline
\end{tabular}

Note-Because of space limitations, the table presents only the first 10 eigenvalues, but only the first $p / 2$ values are usually needed.

chological literature rarely reports the application of the PA technique. It is hoped that the interactive program described herein will ameliorate this problem.

The goals of the present article were twofold: (1) to increase the general level of knowledge concerning PA and (2) to provide a PA computer program. In addition, we provided tabled values that may be used to ensure that the program runs correctly and as references of estimated eigenvalues based on a range of $N$ and $p$ combinations commonly found in the psychological literature. Application of the PA program, however, allows researchers greater flexibility and the ability to address $N$ and $p$ combinations not provided in the tables.

While our program was in the process of review, this journal received another article (O'Connor, 2000) describing SAS and SPSS programs for accomplishing PA as well as similar programs for Velicer's (1976) minimum average partial (MAP) test. We and O'Connor both recognized that while various statistical articles have documented the advantages of PA and MAP relative to the older procedures used in statistical program packages, these superior procedures were seldom used in published exploratory factor analysis. We both felt that a probable cause for the disparity between theory and practice was the lack of modern PC-compatible software to accomplish the more modern procedures. O'Connor's approach was to employ the computing power of SPSS and SAS packages, whereas we provide a PC-compatible stand-alone program in FORTRAN. Both provide basically the same answers for PA, although ours is a bit faster, has interactive parameter specification, and provides only mean values because computation of percentiles requires storage and sorting, which is both space and time-consuming; such computations could be easily added to the present software.

\section{Program Availability}

The PA program software may be obtained by sending a PC-formatted 3.5-in. disk together with a selfaddressed, stamped disk mailer addressed to the authors at 2007 Percival Stern Hall, Department of Psychology, Tulane University, New Orleans, LA 70118-5698. J.D.K. may also be reached by e-mail at jkaufma@mailhost. tcs.tulane.edu, and the program may be obtained by email attachment as an executable file or downloaded from the following web site: http://studentweb.tulane. edu/ jkaufma. The PA program is capable of running on Windows 95 and higher.

\section{REFERENCES}

Allen, S. J., \& Hubbard, R. (1986). Regression equations for the latent roots of random data correlation matrices with unities on the diagonal. Multivariate Behavioral Research, 21, 393-398.

CATtEll, R. B. (1966). The scree tests for the number of factors. Multivariate Behavioral Research, 1, 245-276.

Comrey, A. L., \& LEE, H. B. (1992). A first course in factor analysis. Hillsdale, NJ: Erlbaum.

Cota, A. A., Longman, R. S., Holden, R. R., Fekken, C., \& Xinaris, S. (1993). Interpolating 95 th percentile eigenvalues from random data: An empirical example. Educational \& Psychological Measurement, 53, 585-596.

EveritT, B. S. (1975). Multivariate analysis: The need for data, and other problems. British Journal of Psychiatry, 126, 237-240.

Fabrigar, L. R., Wegener, D. T., MacCallum, R. C., \& Strahan, E. J. (1999). Evaluating the use of exploratory factor analysis in psychological research. Psychological Methods, 3, 272-299.

HoRN, J. L. (1965). A rationale and test for the number of factors in factor analysis. Psychometrica, 30, 179-185. 
Humphreys, L. G., \& Montanelli, R. G., Jr. (1975). An investigation of the parallel analysis criterion for determining the number of common factors. Multivariate Behavioral Research, 10, 193-205.

International Mathematical and Statistical Libraries (1982). International mathematics and statistical libraries reference manual (9th ed.). Houston: Author.

KAISER, H. F. (1960). The application of electronic computers to factor analysis. Education \& Psychological Measurement, 20, 141-151.

LAUTENSCHLAGER, G. J. (1989). A comparison of alternatives to conducting Monte Carlo analyses for determining parallel analysis criteria. Multivariate Behavioral Research, 24, 365-395.

Lautenschlager, G. J., Lance, C. E., \& Flaherty, V. L. (1989). Parallel analysis criteria: Revised equations for estimating the latent roots of random data correlation matrices. Educational \& Psychological Measurement, 49, 339-345.

LAWLEY, D. N., \& MAXWELl, A. E. (1963). Factor analysis as a statistical method. London: Butterworth.

Longman, R. S., Cota, A. A., Holden, R. R., \& FeKken, G. C. (1989). PAN: A double precision FORTRAN routine for the parallel analysis method in principal components analysis. Behavior Research Methods, Instruments, \& Computers, 21, 477-480.
MacCallum, R. C., Widaman, K. F., Zhang, S., \& Hong, S. (1999). Sample size in factor analysis. Psychological Methods, 4, 84-99. Montanelli, R. G., JR., \& Humphreys, L. G. (1976). Latent roots of random data correlation matrices with squared multiple correlations on the diagonal: A Monte Carlo study. Psychometrika, 41, 341-348.

O'CONNOR, B. P. (2000). SPSS and SAS programs for determining the number of components using parallel analysis and Velicer's MAP test. Behavior Research Methods, Instruments, \& Computers, 32, 396-402.

Sharma, S. (1996). Applied multivariate techniques. New York: Wiley.

TABAChNick, B. G., \& Fidell, L. S. (1996). Using multivariate statistics. New York: HarperCollins.

Turner, N. E. (1998). The effect of common variance and structure pattern on random data eigenvalues: Implications for the accuracy of parallel analysis. Educational \& Psychological Measurement, 58, $541-568$.

Velicer, W. F. (1976). Determining the number of components from the matrix of partial correlations. Psychometrika, 41, 321-327.

ZWICK, W. R., \& VeLiCER, W. F. (1986). Comparison of five rules for determining the number of components to retain. Psychological Bulletin, 99, 432-442.

\section{APPENDIX}

c Expected eigenvalues (for parallel analysis)

c uses "rnset, rnnof, rnnoa, corvc, \& evasf" from IMSL

c

use numerical_libraries

dimension $x(50), \operatorname{dat}(1000,50), \mathrm{r}(50,50), \operatorname{inc}(1,1), \mathrm{e}(50), \mathrm{al}(50)$

logical sml

sml $=$. false.

c reset the random number generator $\&$ discard 1 st random number

call rnset $(0)$

$\mathrm{gb}=\mathrm{rnnof}()$

c set the number of iterations

nitr $=1000$

xitr=nitr

c enter parameters

1 write $\left.{ }^{*}, 2\right)$

2 format $(/ /$ Enter no. variables, no. subjects $(0,0$ to stop) ',\$)

$\operatorname{read}(*, *) \mathrm{m}, \mathrm{n}$

if(m.le.0.or.n.le.0)stop

if(m.le.50.and.n.le.1000)goto 4

write $(*, 3)$

3 format (' Problem exceeds current maximum of 50 variables'/

+ 'or 1000 subjects')

stop

4 continue

do $5 \mathrm{i}=1, \mathrm{~m}$

al $(\mathrm{i})=0.0$

5 continue

c start iterations here

do $8 \mathrm{k}=1$, nitr

do $6 \mathrm{i}=1, \mathrm{n}$

call rnnoa(m,x)

do $6 \mathrm{j}=1, \mathrm{~m}$

$\operatorname{dat}(\mathrm{i}, \mathrm{j})=\mathrm{x}(\mathrm{j})$

6 continue

call corvc $(0, n, m$, dat $, 1000,0,0,0,2, x, r, 50$, inc $, 1, n o, n m, g b)$ 


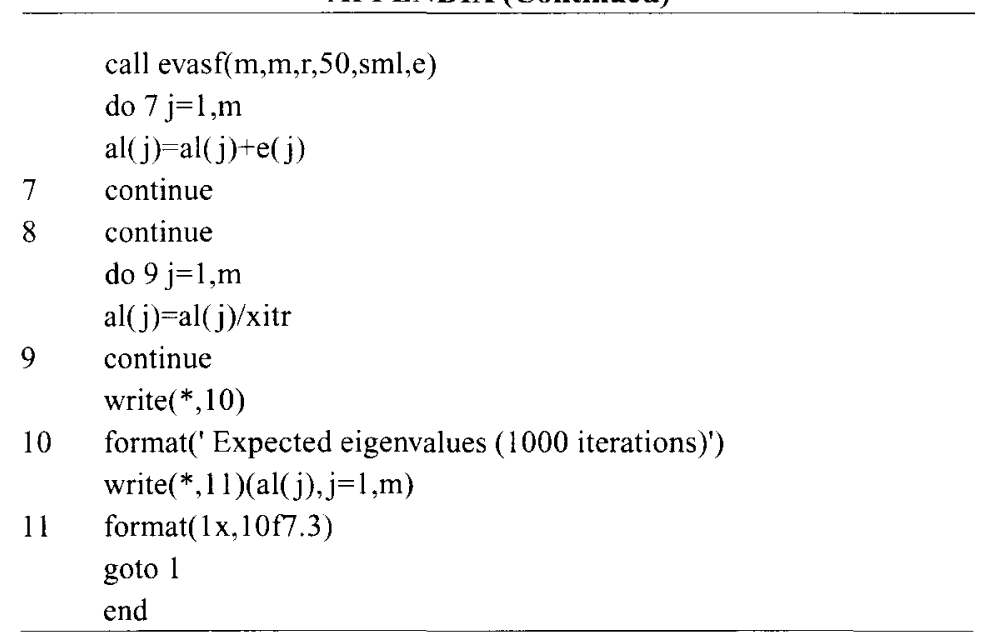

(Manuscript received May 28, 1999;

revision accepted for publication March 12, 2000.) 